\title{
6 Die geschlossenen Venerologischen Stationen in Berlin und Berlin-Buch
}

\subsection{Von den dezentralen Zwangseinweisungen zur geschlossenen Venerologischen Station im Krankenhaus Prenzlauer Berg}

Die Stadt Berlin hatte nach dem Ende des Zweiten Weltkriegs einen besonderen Status. Die ehemalige Reichshauptstadt wurde in vier Sektoren geteilt und von der Alliierten Kommandantur verwaltet. Dieses Organ, bestehend aus den vier Besatzungsmächten USA, Großbritannien, Frankreich und Sowjetunion, übte gemeinsam die Kontrolle über Groß-Berlin aus und war dem Alliierten Kontrollrat unterstellt, der die Kontrollfunktion für Deutschland wahrnahm. In Groß-Berlin regierte die Alliierte Kommandantur in Form von „Anordnungen an den Berliner Magistrat und an den Oberbürgermeister". Diese mussten einstimmig von den Kommandanten der vier Besatzungsmächte beschlossen werden. Eine solche Anordnung für Groß-Berlin war die der „Alliierten Kommandantur Berlin BK/O (46) 257 und 298 über Bekämpfung von Geschlechtskrankheiten vom 7. Juni bzw. 9. Juli 1946“. Die darin festgeschriebenen Regelungen entsprachen jenen Maßnahmen, die im SMAD-Befehl Nr. 25 zur Bekämpfung von Geschlechtskrankheiten in der SBZ festgelegt waren (Kap. 3.1). Der Berliner Magistrat konzentrierte sich bei der Umsetzung der Anordnung vor allem auf zwei Bereiche: die Einrichtung von Beratungs-, Behandlungsund Fürsorgestellen für die Versorgung Geschlechtskranker sowie die Einrich- 
tung polizeilich überwachter Stationen für die Behandlung von Syphilis- und Gonorrhoe-Patienten. ${ }^{483}$

Der flächendeckende Auf- und Ausbau von „Bezirksbehandlungs- und Bezirksfürsorgestellen für die Bekämpfung von Geschlechtskrankheiten“ wurde dezentral umgesetzt. Diese Fürsorgestellen entstanden in fast allen Bezirken von Groß-Berlin und waren für die Betreuung von geschlechtskranken Menschen zuständig ${ }^{484}$ und nahmen vor allem im sowjetischen Sektor noch im Jahr 1946 in den jeweiligen Stadtbezirken (Pankow, Weißensee, Prenzlauer Berg, Mitte, Friedrichshain, Lichtenberg, Köpenick und Treptow) die Arbeit auf. ${ }^{485}$ Ihre Aufgabe bestand einerseits in der Prävention. So klärten sie die Bevölkerung mit Vorträgen, Plakaten, Broschüren oder Flugblättern auf. Andererseits betreuten sie geschlechtskranke Personen: Sie hielten Kontakt zu Prostituierten oder Menschen mit HwG-Verdacht, führten die ambulante Behandlung von Geschlechtskrankheiten durch und waren für Hospitalisierungen und Zwangseinweisungen verantwortlich. Koordiniert wurden diese dezentralen Einrichtungen im sowjetischen Sektor durch die „Zentrale Behandlungs- und Beratungsstelle für die Bekämpfung von Geschlechtskrankheiten“".486 Zudem erhob und sammelte die Zentrale statistische Daten für alle Stadtbezirke, überwachte Prostituierte und HwG-Personen, steuerte die Umsetzung der Anordnungen der Alliierten Kommandantur und die Zusammenarbeit mit entsprechenden Stellen in den westlichen Besatzungszonen.

Ende der 1940er Jahre hatten sich zwei Personengruppen herauskristallisiert, die sich überdurchschnittlich häufig mit einer Geschlechtskrankheit infizierten, so die „Zentrale Behandlungs- und Beratungsstelle für die Bekämpfung von Geschlechtskrankheiten“: Prostituierte sowie Mädchen und junge Frauen im Alter von 18 bis 25 Jahren. ${ }^{487}$ Vor diesem Hintergrund wurde der Plan entwickelt, ein Heim für geschlechtskranke Mädchen und junge Frauen in Berlin-Buch zu eröffnen. In die zwischen 1898 und 1930 entstandene Heilanstalt Berlin-Buch ${ }^{488}$ war im November 1943 die Hautklinik der Charité eingezogen, nachdem die Charité durch einen Bombenangriff zerstört worden war. ${ }^{489}$ Vorläufig wurde die Hautklinik im Haus 305 untergebracht und nach dem Ende des Zweiten Weltkriegs unter der Leitung des Chefarztes Friedrich Jacobi (18941965) im Haus 213 wiedereröffnet. Von 1945 bis 1950 bestanden im Haus 213 zwei

483 LA Berlin: C Rep. 118 - Magistrat von Berlin, Abteilung Gesundheits- und Sozialwesen. Nr. 863: Verordnung zur Bekämpfung der Geschlechtskrankheiten 1955-1956. Die Anordnung der Alliierten Kommandantur Berlin BK/0 (46) 257 und 298 über Bekämpfung von Geschlechtskrankheiten vom 7. Juni bzw. 9. Juli 1946 (VOBI. 358), S. 1.

484 LA Berlin: C Rep. 118 - Magistrat von Berlin, Abteilung (...). Die Anordnung (Anm. 483).

485 LA Berlin: C Rep. 118 - Magistrat von Berlin, Abteilung Gesundheits- und Sozialwesen. Nr. 1084: Struktur-, Stellenplan- und Geschäftsverteilungsunterlagen der Zentralstelle zur Bekämpfung der Geschlechtskrankheiten (1946-1952).

486 LA Berlin: C Rep. 118 - Magistrat von Berlin, Abteilung (...). Geschäftsverteilungsunterlagen (Anm. 485).

487 LA Berlin: C Rep. 118 - Magistrat von Berlin, Abteilung Gesundheits- und Sozialwesen. Nr. 249: Statistische Jahresberichte 1949 und 1951 über Neuerkrankungen an Geschlechtskrankheiten.

488 Wolff HP, Kalinich A (2006) Zur Geschichte der Krankenhausstadt Berlin-Buch. Mabuse-Verlag Frankfurt am Main, S. 22-62.

489 Harnack K (2000) Die Hautklinik der Charité und die Dermatologie in Berlin (1710-1999). BMV Berlin, S. 115. 
Abteilungen für Gonorrhoe-Patientinnen. ${ }^{490}$ Vor diesem Hintergrund wurde geplant, ein Heim für geschlechtskranke Mädchen in Berlin-Buch zu eröffnen. Dort sollten „a) Mädchen und Frauen einer Behandlung unterzogen werden“. Darüber hinaus plante man, dass „,b) die Frist zwischen den notwendigen $\mathrm{Ku}$ ren durch einen Aufenthalt ausgefüllt wird, der erzieherische Funktionen erfüllen soll“. Mit der Isolierung in Berlin-Buch sollten die Mädchen und jungen Frauen zum einem von älteren Prostituierten und straffälligen Frauen getrennt und venerologisch behandelt werden. Zum anderen sollten sie pädagogisch betreut werden. Dazu sollten die Mädchen und jungen Frauen über mehrere Wochen - manchmal bis zu drei Monaten - in Berlin-Buch untergebracht, medizinisch versorgt und erzogen werden. ${ }^{491}$

Anfang der 1950er Jahre gingen die Infektionsraten zurück. Vor diesem Hintergrund wurde im sowjetischen Sektor Berlins eine stärkere Zentralisierung der bisher dezentral organisierten „Bezirksbehandlungs- und Bezirksfürsorgestellen“ beschlossen. Aus den ehemals acht Stellen wurden durch Zusammenlegung vier. Gleichzeitig musste die „Zentrale Behandlungs- und Beratungsstelle zur Bekämpfung der Geschlechtskrankheiten“ von Berlin-Mitte in die bisherige „Bezirksfürsorgestelle zur Bekämpfung der Geschlechtskrankheiten“ in Berlin-Prenzlauer Berg umziehen und in der Nordmarkstraße 15 Quartier nehmen. ${ }^{492}$ Dort wurde sie zusammen mit der geschlossenen Venerologischen Station in einem Teil des Krankenhauses Prenzlauer Berg in der heutigen Fröbelstraße untergebracht.

Das Krankenhaus Prenzlauer Berg war zwischen 1886 und 1889 von dem Berliner Architekten Hermann Blankenstein (1829-1910) als Friedrich-WilhelmHospital Fröbelstraße errichtet worden. Es bestand ursprünglich aus Verwaltungs- und Hospitalgebäuden sowie zwei Siechenhäusern. Teil des Komplexes war die sogenannte „Palme“, die in 40 Baracken Schlafsäle für Obdachlose bereitstellte. ${ }^{493}$ Die Baracken waren ,fischgrätenartig an durchgehenden Fluren angeordnet. Diese mündeten in einem Kopfbau, wo sich Aufnahme und Polizeizimmer, große Brauseanlagen, Aborte und Aufenthaltsräume für die Wärter befanden. Die Beleuchtung erfolgte ausschließlich über die kleinen Glasfenster in den Sheddächern. "494 Bis 1951 wurde der gesamte Gebäudekomplex zurückgebaut. Lediglich drei Baracken mit den Schlafsälen blieben er-

490 Harnack: Die Hautklinik der Charité und die Dermatologie (Anm. 489).

491 LA Berlin: C Rep. 118 - Magistrat von Berlin, Abteilung Gesundheits- und Sozialwesen. Nr. 421. Bekämpfung der Geschlechtskrankheiten 1946-1953. Kurzbericht zur Einrichtung eines Heims für geschlechtskranke Mädchen in Berlin Buch (1948).

492 LA Berlin: C Rep. 118 - Magistrat von Berlin, Abteilung Gesundheits- und Sozialwesen. Nr. 1084: Struktur-, Stellenplan- und Geschäftsverteilungsunterlagen der Zentralstelle zur Bekämpfung der Geschlechtskrankheiten (1946-1952). Zentralstelle zur Bekämpfung der Geschlechtskrankheiten (einschließlich der Zentral- Behandlungsstelle) Fischerstraße 37-42.

493 Jakoby B (2006) Das Hospital und Siechenheim Fröbelstraße (1889-1934). In: Berlin-Brandenburgische Geschichtswerkstatt (Hrsg.) Prenzlauer, Ecke Fröbelstrasse. Hospital der Reichshauptstadt, Haftort der Geheimdienste, Bezirksamt Prenzlauer Berg 1889-1989. 25-49. Lukas-Verlag Berlin, hier: S. 27.

494 Lemke E, Grünke C (1995) Festschrift zum 55jährigen Bestehen des Krankenhauses Prenzlauer Berg. Krankenhaus Prenzlauer Berg Berlin, S. 6. 
halten und wurden zur geschlossenen Venerologischen Station im Krankenhaus Prenzlauer Berg (Station 11) umgebaut (Abb. 16 und 17). Im Juli 1952 waren wesentliche Teile der Umbauarbeiten abgeschlossen. Nur die Gartenanlagen für die geschlossene Venerologische Station waren noch nicht fertiggestellt. Jedoch war dieser Bereich für das Funktionieren der Station notwendig. Denn besonders während der heißen Jahreszeiten war „eine Belegung der Station ohne Auslaufmöglichkeit im Gartengelände kaum vorstellbar“.495

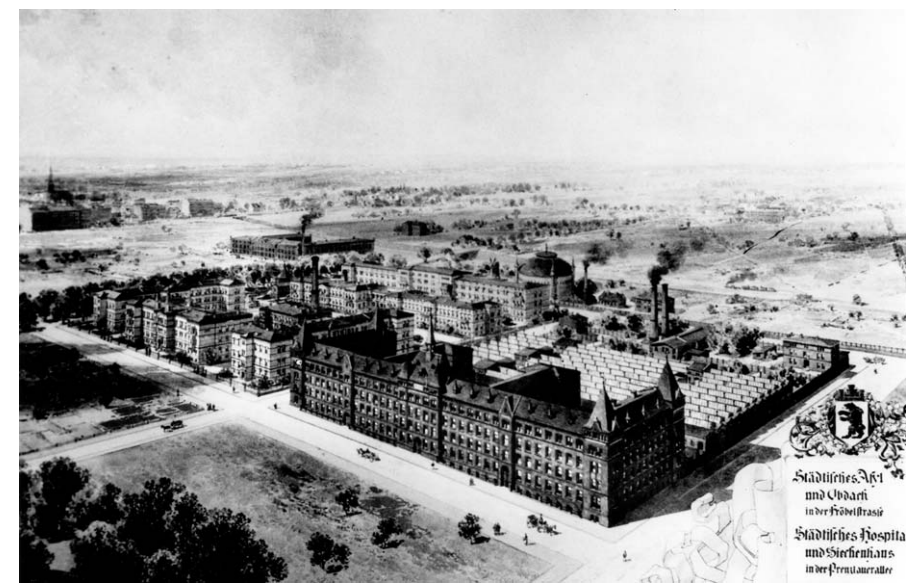

Abb. 15 Obdachlosenheim „Die Palme“ (um 1920)

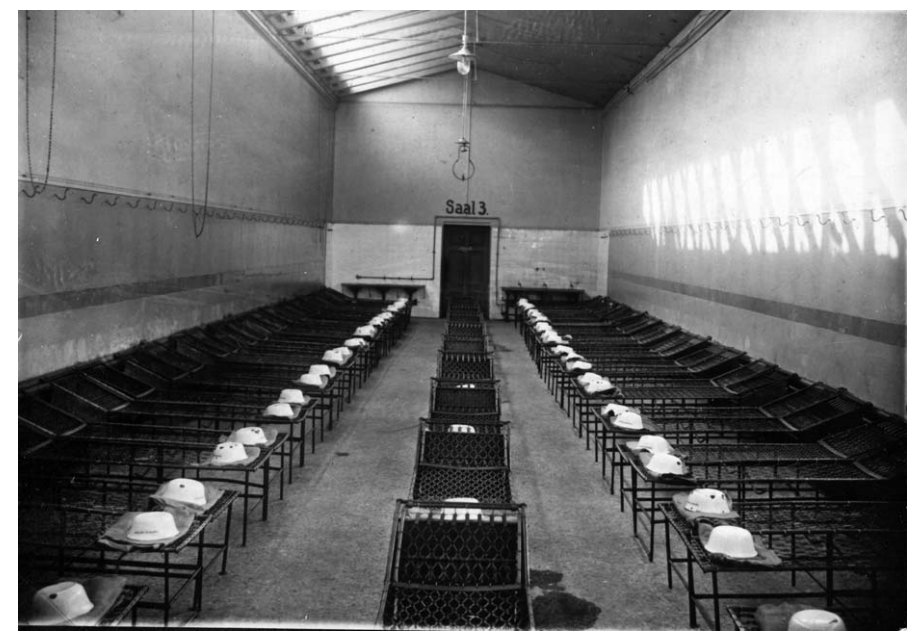

Abb. 16 Blick in einen Schlafsaal des Obdachlosenheims (um 1920)

495 LA Berlin: C Rep. 118 - Magistrat von Berlin, Abteilung Gesundheits- und Sozialwesen. Nr. 421. Bekämpfung der Geschlechtskrankheiten 1946-1953. An die Krankenhausverwaltung Prenzlauer Berg. Berlin, den 21. Juli 1952. 
Von ihrem neuen Sitz aus koordinierte die „Zentrale Behandlungs- und Beratungsstelle“ seit 1952 die vier zusammengelegten „Bezirksbehandlungs- und Bezirksfürsorgestellen“. ${ }^{496}$ Zusätzlich zur Zusammenlegung mussten die vier verbliebenen Stellen Teile ihrer bisherigen Aufgaben an die zentrale Stelle abtreten. Künftig sollte die Betreuung von Prostituierten und HwG-Personen ausschließlich durch die „Zentrale Behandlungs- und Beratungsstelle“ in der Nordmarkstraße 15 umgesetzt werden. Gleichzeitig wurde den „Bezirksbehandlungs- und Bezirksfürsorgestellen“ zum 1. Januar 1953 untersagt, Zwangseinweisungen zu veranlassen. Künftig sollten solche Einweisungen nur noch durch die „Zentrale Behandlungs- und Beratungsstelle“ koordiniert werden. ${ }^{497}$ Seit dieser Zeit gab es Zwangseinweisungen im sowjetischen Sektor Berlins in der geschlossenen Venerologische Station des Krankenhauses Prenzlauer Berg.

\subsection{Die geschlossene Venerologische Station im Krankenhaus Prenzlauer Berg}

Zumindest einen indirekten Einblick in die Baracken und den Alltag der dort zwangseingewiesenen Mädchen und Frauen bieten zwei Berichte aus den 1950er Jahren. Der erste Bericht wurde retrospektiv von einer Lehrerin aus einem Berliner Jugendwerkhof verfasst und beschreibt, wie sie ein Mädchen im Krankenhaus Prenzlauer Berg abliefert: „Völlig fremd war mir die Triebhaftigkeit mancher Mädchen. (...) Es waren immer welche darunter, die ausbüchsten (sic!) und sich mit Jungs herumtrieben. Meistens kamen sie nicht weit, weil sie keinen Ausweis hatten. Früher oder später wurden sie von der Polizei wieder zurückgebracht. Wir mussten auf der Hut sein, damit wir uns nicht irgendwelche Krankheiten einfingen. Deshalb schickten wir die Ausreißerinnen zur Untersuchung ins Krankenhaus für Geschlechtskrankheiten an der Nordmarkstraße (heute Fröbelstraße) in Prenzlauer Berg. (...) Einmal fuhr ich selbst mit, weil ich wissen wollte, was sich dort abspielte. Mir war es recht, dass die Krankenschwestern nicht sehr sanft mit den Mädchen umsprangen. Sie sollten ruhig spüren, dass das Ausbüchsen (sic!) nicht nur ein Spaß war. Nach der Untersuchung war das Mädchen eingeschüchtert. ,Warum türmst du auch? Warum machst du so etwas Dämliches? Wenn du eine Krankheit einschleppst, müssen wir alle darunter leiden', versuchte ich ihr die Augen zu öffnen." ${ }^{498}$ Diese kurze Episode liest sich wie die Berichte von Zeitzeugen, die in Halle (Saale) oder Leipzig-Thonberg zwangseingewiesen waren. Sogenannte Herumtreiberinnen wurden von Mitarbeitern des Jugendwerkhofes für ihr Handeln bestraft, indem sie einer Station für Geschlechtskrank-

496 LA Berlin: C Rep. 118 - Magistrat von Berlin, Abteilung (...). Zentralstelle zur Bekämpfung (Anm. 492).

497 LA Berlin: C Rep. 118 - Magistrat von Berlin, Abteilung Gesundheits- und Sozialwesen. Nr. 421. Bekämpfung der Geschlechtskrankheiten 1946-1953.

498 Bigalke-Zell A (2006) „Mach was aus dir!“ Eine Lehrerin erzählt aus ihrem Leben. Rohnstock-Biografien Berlin, S. 88 . 
heiten zugeführt wurden. Die medizinisch-pflegerische Versorgung der Mädchen hatte traumatische Folgen für die Eingewiesenen.

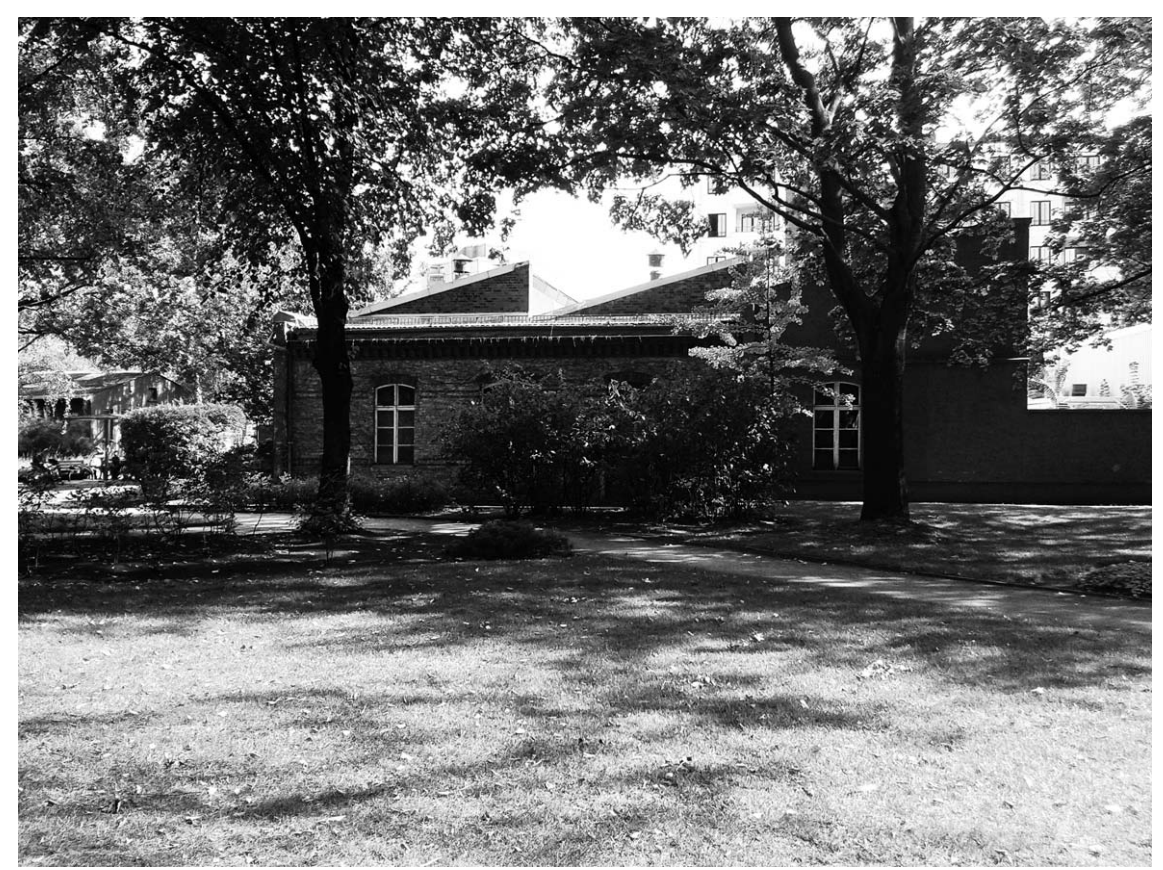

Abb. 17 Baracken mit verschlossenen Shed- bzw. Sägezahndächern der geschlossenen Venerologischen Station im Stadtkrankenhaus Prenzlauer Berg (2015)

Auch ein zweiter Bericht über die geschlossene Venerologische Station in der Nordmarkstraße zeigt Ähnlichkeiten zu Halle (Saale) oder Leipzig-Thonberg. In einem Schreiben des Chefarztes der Station 11 vom 8. Juli 1952 heißt es: „Bei der Zwangsstation im städtischen Krankenhaus Prenzlauer Berg in den drei Baracken des ehemaligen Obdachlosenheimes ,Die Palme“ wird in zunehmenden Maße festgestellt, daß die Patienten sich auf die hölzerne Verschalung der Aborte an der Nordseite der Baracken setzen und das Gesicht und andere unerwünschte Körperteile an das Gitter gegen die Straße drücken, von dort Gespräche mit Bekannten führen und auch anscheinend spielende Kinder dazu anhalten, kleine Besorgungen zu erledigen, Lesematerial von Fenster zu Fenster zu reichen usw. Es werden auch mit den Kindern Scherze getrieben, die für den kindlichen Entwicklungsstand als ungeeignet angesehen werden müssen. “499 In Berlin wie in Halle (Saale) versuchten zwangseingewiesene Frauen, hinter vergitterten Fenstern Kontakt zur Außenwelt aufzunehmen,

499 LA Berlin: C Rep. 118 - Magistrat von Berlin, Abteilung Gesundheits- und Sozialwesen. Nr. 421. Bekämpfung der Geschlechtskrankheiten 1946-1953. Ges. I/21. Berlin, den 8. Juli 1952. 
tauschten Informationen mit Angehörigen aus oder kommunizierten mit anderen Zwangseingewiesenen. Um die Kommunikation mit der Außenwelt zu unterbinden, wurde in einem weiteren Schreiben festgelegt, dass der „beantragte Einbau von Glasbausteinen in den Toiletten der Station " als vordringlich erscheint, „da die dauernden Gespräche der Patientinnen mit Besuchern und Jugendlichen aus den Straßen eine Möglichkeit der Zersetzung der Moral unserer Jugendlichen bieten“. ${ }^{500}$

Die anfänglichen baulich bedingten Schwierigkeiten wurden überwunden und selbst die Gartenanlagen für den Freigang der Zwangseingewiesenen wurden fertiggestellt. Die Nordmarkstraße 15 etablierte sich als „Zentrale Behandlungs- und Beratungsstelle für die Bekämpfung von Geschlechtskrankheiten “ mit ihrer zentral eingerichteten geschlossenen Venerologischen Station in Ostberlin. Dies geht unter anderem aus einem Bericht des Jahres 1963 hervor. Darin werden die Durchsetzung der „Verordnung zur Bekämpfung der Geschlechtskrankheiten" von 1961 und die damit verbundenen offenen Fragen angesprochen. ${ }^{501}$ Uneindeutig war etwa die Unterstützung durch die Volkspolizei bei der Zuführung von Geschlechtskranken, die in der Verordnung angesprochen wurde (Kap. 3.4). In diesem Zusammenhang wird noch einmal ausdrücklich die Notwendigkeit einer geschlossenen Venerologischen Station betont: „Die geschlossene Abteilung für Ge[schlechts]-Kranke, die sich in unmittelbarer Nähe der Zentralstelle befindet, und aus technischen Gründen auch dort untergebracht sein muss, ist nach wie vor unbedingt erforderlich." 502

Ende der 196oer Jahre zog die „Zentralstelle zur Bekämpfung der Geschlechtskrankheiten " von der Nordmarkstraße in die nahegelegene Christburger Straße und die geschlossene Venerologische Station des Krankenhauses Prenzlauer Berg wurde 1968 dem Städtischen Klinikum Berlin-Buch zugeordnet. Sie umfasste zu dieser Zeit „29 Betten (20 Frauen- und 9 Männerbetten) und verfügte über einen geschlossenen Garten, der von den Kranken am Tage zu bestimmten Zeiten für Freigänge genutzt werden konnte“. ${ }^{503}$ Bauliche Mängel an den Baracken führten im Jahr 1971 zur Auflösung der geschlossenen Venerologischen Station, „die sich in den Baracken in Höhe des heutigen Küchenhauses befand, und in der geschlechtskranke Frauen, die sich nicht freiwillig einer Behandlung unterzogen, unter unwürdigen Bedingungen behandelt wurden“. ${ }^{504} \mathrm{Da}$ -

500 LA Berlin: C Rep. 118 - Magistrat von Berlin, Abteilung Gesundheits- und Sozialwesen. Nr. 421. Bekämpfung der Geschlechtskrankheiten 1946-1953. An die Krankenhausverwaltung Prenzlauer Berg. Berlin, den 21.7.1952.

501 LA Berlin: C Rep. 118 - Magistrat von Berlin, Abteilung Gesundheits- und Sozialwesen. Nr. 935: Durchsetzung der V0 zur Bekämpfung der Geschlechtskrankheiten vom 23. Januar 1961 in Berlin. Bericht der Zentralstelle zur Bekämpfung der Geschlechtskrankheiten Berlin N0 55, Nordmarkstraße 15, Eingang Diesterweg vom 14. September 1963.

502 LA Berlin: C Rep. 118 - Magistrat von Berlin, Abteilung (...). Bericht der Zentralstelle (Anm. 501), S. 3.

503 LA Berlin: C Rep. 118 Nr. 909 1977. Reorganisation und Rekonstruktion der Psychiatrischen Klinik im Städtischen Klinikum Buch. Bericht im Zusammenhang mit den „besonderen Vorkommnissen“ in der geschlossenen Abteilung der Hautklinik des Städtischen Klinikum Berlin-Buch, Haus 114, Station C, Berlin, den 8. August 1977.

504 Lemke, Grünke: Festschrift zum 55jährigen Bestehen des Krankenhauses (Anm. 494), S. 15. 
mit endete die Praxis der Zwangseinweisung von krankheitsverdächtigen Personen in Berlin nicht, sondern die bisher im Krankenhaus Prenzlauer Berg zwangseingewiesenen Frauen wurden in Berlin-Buch aufgenommen. Hierzu wurde im Dezember 1971 in Berlin-Buch „eine geschlossene Abteilung für ,weibliche uneinsichtige Gefährdete und dringend krankheitsverdächtige Personen` mit 11 Betten eröffnet, nachdem die gleiche Abteilung für Männer und Frauen im Krankenhaus Prenzlauer Berg geschlossen worden war". ${ }^{505}$ Hier zeigt sich das Nahtstück zwischen den geschlossenen Venerologischen Abteilungen in Berlin-Prenzlauer Berg und Berlin-Buch. Die im Krankenhaus Prenzlauer Berg aufgelöste Station wurde im Krankenhaus Berlin-Buch übergangslos fortgeführt. Die Praxis der Zwangseinweisung wurde nicht aufgehoben, sondern an einem anderen Ort kontinuierlich fortgesetzt.

\subsection{Die geschlossene Venerologische Station im Städtischen Krankenhaus Berlin-Buch}

Als die geschlossene Venerologische Station des Krankenhauses Prenzlauer Berg nach Berlin-Buch verlagert wurde, konnte sie in eine bereits etablierte Struktur integriert werden. 1943 war die Hautklinik der Charité nach BerlinBuch verlagert worden und hatte im Haus 213 zwei Abteilungen für ConorrhoePatientinnen. 1951 wurde die Hautklinik im Haus 114 unter der Leitung von Karl Linser eingeweiht (Kap. 3.4). Als Linser 1956 die neu eingeweihte Hautklinik an der Charité übernahm, erhielt Dr. med. Erich Thiel die Leitung der Hautklinik in Berlin-Buch, die er bis 1961 innehatte. ${ }^{506} 1962$ wurde Dr. med. Rolf Stöps (1919-1968), Oberarzt bei Karl Linser an der Charité, die Leitung in Berlin-Buch übertragen. Stöps hatte sich 1963 mit der Arbeit Das H-Anti-H-Problem in der Dermatologie ${ }^{507}$ habilitiert. Als er 1967 eine Professur an der MartinLuther-Universität Halle-Wittenberg erhielt, folgte ihm Dr. med. Günter Elste als Chefarzt der Hautklinik in Berlin-Buch. ${ }^{508}$ Auch Elste war langjähriger Oberarzt bei Linser an der Berliner Charité-Hautklinik. Obwohl er nicht habilitierte, zählte er zu den führenden Venerologen der DDR, der auch international bekannt wurde. Er verfasste seit 196o das Kapitel „Geschlechtskrankheiten“ im Statistischen Jahrbuch der DDR und war Autor im Lehrbuch Dermatologie. Ein Lehrbuch für Studenten, das in mehreren Auflagen erschien. ${ }^{509} 1972$ wurde er in das Exekutivkomitee der „Internationalen Union zur Bekämpfung der Geschlechtskrankheiten und Treponematosen " aufgenommen und vertrat die DDR seit 1975 im „Expert Panel on Bacterial and Venereal Infections“ bei

505 Harnack: Die Hautklinik der Charité und die Dermatologie (Anm. 489), S. 116. 506 Harnack: Die Hautklinik der Charité und die Dermatologie (Anm. 489), S. 116.

507 Stöps R (1963) Das H-Anti-H-Problem in der Dermatologie. Med. Diss., Berlin.

508 Harnack: Die Hautklinik der Charité und die Dermatologie (Anm. 489), S. 116.

509 Braun W (Hrsg.) (1975) Dermatologie. Ein Lehrbuch für Studenten. Verlag Volk und Gesundheit Berlin. 
der Weltgesundheitsorganisation (WHO). ${ }^{510}$ Unter seiner Leitung wurde die „Zentralstelle zur Bekämpfung der Geschlechtskrankheiten“ in der Berliner Christburger Straße an die Hautklinik von Berlin-Buch angegliedert. ${ }^{51}$

Durch die Angliederung war die Zentralstelle der Knotenpunkt, über den die Einweisungen nach Berlin-Buch liefen. Die Mitarbeiter sollten die als Infektionsquelle gemeldeten Personen mit drei Anschreiben und einem Hausbesuch kontaktieren und die krankheitsverdächtigen Personen zur Untersuchung auffordern. Kamen die Krankheitsverdächtigen dem nicht nach, erfolgte zunächst die Einweisung auf eine offene Station. Widersetzten oder entzogen sie sich der Einweisung, so sollte die Zwangseinweisung in die geschlossene Venerologische Station des Städtischen Krankenhauses Berlin-Buch greifen. Waren Personen nicht auffindbar, wurden sie zur Fahndung ausgeschrieben. Weiterhin nahm man alle Personen fest, die aufgegriffen wurden, etwa durch die Kriminalpolizei, Transportpolizei, Abteilung Inneres oder die Jugendfürsorge-Heimerziehung, und die als „Ausreißer aus Jugendwerkhöfen, Kinderheimen, Elternhaus“ galten, „aber auch Erwachsene, die sich, die Anonymität der Hauptstadt nutzend, als Stadtstreicher ohne festen Wohnsitz und Arbeit“ herumtrieben. ${ }^{512}$ Sie wurden der „Zentralstelle zur Bekämpfung der Geschlechtskrankheiten“ vorgestellt, wenn ein Verdacht auf „HwG-Unwesen“, ein Krankheitsverdacht oder eine Meldung als Infektionsquelle vorlag.

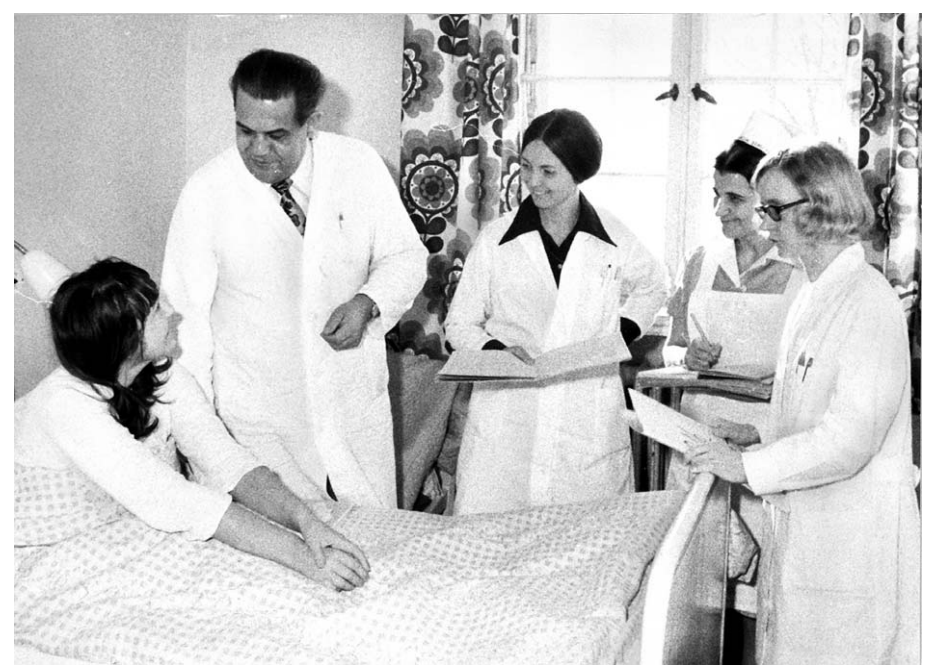

Abb. 18 Günter Elste (um 1970)

510 Wolff, Kalinich: Zur Geschichte der Krankenhausstadt Berlin-Buch (Anm. 488), S. 238.

511 Wolff, Kalinich: Zur Geschichte der Krankenhausstadt Berlin-Buch (Anm. 488), S. 235.

512 LA Berlin: C Rep. 118 Nr. 909 1977. Reorganisation und Rekonstruktion der Psychiatrischen Klinik im Städtischen Klinikum Buch. Bericht im Zusammenhang mit (Anm. 503), S. 2. 
Im Haus 213, in dem seit Ende des Zweiten Weltkriegs zwei Abteilungen für Gonorrhoe-Kranke untergebracht waren, hatte man in den 197oer Jahren sogenannte „,kriminell gewordene und psychisch Kranke und kriminell gewordene Täter im Sinne der Untersuchung auf Zurechnungsfähigkeit, Haftfähigkeit und Geisteszustand stationär untergebracht“, wie aus einem Schreiben an den Minister für Gesundheitswesen, Dr. med. Ludwig Mecklinger (19191994) vom 30. November 1977 hervorgeht. ${ }^{513}$ Im Haus 114, das 1951 in Berlin-Buch als Hautklinik eröffnete wurde, zog 1971 die geschlossene Venerologische Station ein. In dem Schreiben an den Gesundheitsminister findet sich auch ein Abschnitt zur geschlossenen Venerologischen Abteilung in Berlin-Buch: „Die auf der Station $114 \mathrm{C}$ (geschlossene Abteilung) zur Aufnahme gelangenden weiblichen Personen sind keinesfalls ,kriminell gewordene“ psychisch Kranke, sondern überwiegend zur Asozialität neigende Jugendliche, die sich den gesetzlichen Verpflichtungen zur Behandlung und Nachkontrolle einer Geschlechtskrankheit entziehen bzw. nicht nachkommen." 514

Die geschlossene Station 114 C wurde 1971 als ,eine improvisierte geschlossene Abteilung mit 11 Frauenbetten" eingerichtet, wie der Chefarzt Elste in einem Bericht vom 5. August 1977 zur Situation auf der Station ausführt. ${ }^{15}$ Was Elste unter ,improvisiert“ verstand, wird im Weiteren deutlich. „Daselbst wurden die normalen Klinikfenster einer im ersten Stock liegenden verschließbaren Abteilung einer Frauenstation abgeschlossen, die Krankenzimmer mit normalem Krankenhausmobiliar ausgestattet und mit der Arbeit begonnen. "Die geschlossene Station hatte drei nicht abschließbare Krankenzimmer, die mit „Krankenhaus-Normalbetten“, Nachtschränken, Tischen, Hockern und Stühlen ausgestattet waren. In der Abteilung befanden sich ein Wasserklosett und eine Badewanne. „Freistunden auf einem ungesicherten Balkon“ scheiterten daran, „daß häufig pro Schicht nur eine Schwester oder Hilfskraft für die geschlossene Abteilung einschließlich 36 weiblicher Hautkranken der offenen Station zur Verfügung" stand. ${ }^{516}$

Eine Zeitzeugin, die im August 1975 auf der geschlossenen Station 114 C zwangseingewiesen war, beschreibt die Einweisung und die Station: „Ich habe im Arbeiterwohnheim übernachtet. Ich war noch nicht 16, wurde dann von der Heimleitung als nicht angemeldeter Übernachtungsgast, der minderjährig war, der Polizei übergeben und habe dann wirklich von Vormittag bis ca. 18 Uhr, schätze ich, auf der Polizeiwache gesessen, ohne zu wissen, was jetzt

513 LA Berlin: C Rep. 118 Nr. 909 1977. Reorganisation und Rekonstruktion der Psychiatrischen Klinik im Städtischen Klinikum Buch. Einschätzung über Betreuung „kriminell gewordener“ und psychisch Kranker in psychischen Einrichtungen. Berlin, den 3011.1977, S. 3.

514 LA Berlin: C Rep. 118 Nr. 909 1977. Reorganisation und Rekonstruktion (...). Einschätzung über Betreuung „kriminell gewordener“ (Anm. 513), S. 2.

515 LA Berlin: C Rep. 118 Nr. 909 1977. Reorganisation und Rekonstruktion (...). Einschätzung über Betreuung „kriminell gewordener“ (Anm. 513), S. 3.

516 LA Berlin: C Rep. 118 Nr. 909 1977. Reorganisation und Rekonstruktion (...). Einschätzung über Betreuung „kriminell gewordener“ (Anm. 513), S. 4. 
passiert. Wurde auch da schlecht behandelt, also zur Toilette ist einer mitgekommen, Tür musste offen bleiben, also wie so ein Schwerverbrecher, nichts $\mathrm{zu}$ trinken, nichts zu essen und habe gewartet, dass meine Mutter mich abholt. Und die kam dann auch, hat mit mir aber gar nicht gesprochen. Also ich saß im Flur an einer Stelle, die sich hinter der Tür befand, also wo dann die Polizisten saßen, wo meine Mutter reinging, dann wurde kurz gesprochen. Ich habe nur irgendwie gehört, ,Wollen Sie mitkommen?" ,Nein, (...) fahren jetzt nach Hause.' Ja und dann war die weg und ich wurde dann ins Polizeiauto gesetzt. Habe dann beim nächsten Mal wieder gefragt, ,Wo geht es denn jetzt hin und wo bringen Sie mich denn hin?', und ja, also, haben sich über mich lustig gemacht und ,Sie kommen jetzt in den Werkhof oder ins Gefängnis, wie sich das für böse Mädchen und Nutten gehört', irgendwie so etwas in der Richtung, ja. Und ja dann waren wir eine ganze Weile unterwegs und dann habe ich halt gesehen, Klinikum Buch sind wir reingefahren, ein großes Schild und dann auf dem Gelände haben wir vor einem Haus gehalten und da wurde ich dann reingebracht, im Untersuchungszimmer und habe dann gefragt, warum ich denn da bin und so, Untersuchung. Polizei war dann da weg. Ja und dann ging es gleich los, Sachen ausziehen, alles in einen Beutel, Geld zählen, also ich weiß gar nicht, ob ich überhaupt etwas dabei hatte, keine Ahnung. Jedenfalls wurde mir alles, ich musste mich völlig splitternackt ausziehen, alles abgenommen. Dann habe ich so einen blaugrauen, wie so einen Kasack bekommen, Badelatschen und das war es erst einmal. Ich weiß, dass mir viel Blut abgenommen wurde, da haben mehrere Leute an mir rumgepikst, letztendlich aus der Hand dann und ja, also mir wurde gesagt, dass ich da auf Geschlechtskrankheiten untersucht werde. Mir kam das schon komisch vor, dass mir die Sachen so weggenommen worden sind, aber hab immer noch nicht geschnallt, dass ich dann danach nicht wieder lieben kann. " Die Station war im ersten Stockwerk. Am Ende eines langen Flures war eine „doppelte Eisentür. So wie man die vor große Fahrstühle hat, so ich versuche mich immer daran zu erinnern, ich glaube, da waren auch solche Gucklöcher drinnen. Die hat sie aufgeschlossen und dahinter war dann mit einem Stückchen Abstand ein Gitter, also ein Eisengitter vom Boden bis zur Decke, ja. "Wenn man die Station betrat, „war dann gleich rechts ein größeres Zimmer, gegenüber war ein Raum mit einer Toilette mit einem Waschbecken und gleich neben der Tür ein Fenster, ein bisschen weiter oben." Die Fenster waren nicht vergittert, sondern „undurchsichtig und ja, also mit Metallfäden durchzogen, sodass in dem Glas ein Gitter entstand. (...) Und dann ging es rechts um die Ecke, da war ein bisschen ein längerer Flur und da grenzten dann auf der linken Seite noch eine Einzelzelle, die auch mit Eisentür war, mit so einem Cuckloch und dann mein Zimmer, ein Vierbettzimmer. Ja, was dann gegenüber der Tür ein Fenster hatte und auf der rechten Seite, als wenn da das Gebäude schon zu Ende wäre, weiß ich genau. Ja, der Flur ging hinter der Zimmertür dann noch weiter und da war auch die tägliche Untersuchung. Da war der Vorhang und da stand dann eben der Untersuchungsstuhl und da fand das dann jeden Tag statt, jeden Tag. “ In den Zimmern standen „immer zwei Bet- 
ten nebeneinander und immer eine kleine Lücke dazwischen und dann noch zwei gegenüber“. ${ }^{517}$

Der Alltag auf der Station war durch Langeweile und Monotonie geprägt, wie eine zweite Zeitzeugin beschreibt, die 1975 in Berlin-Buch zwangseingewiesen war: „Man ist aufgestanden, irgendwann ging dann die Tür auf, dann bekamen wir Weißbrot mit Marmelade. Ich weiß nicht mal, ob wir ein Messer bekommen haben. Ich weiß bloß, ich erinnere mich noch dran, dass viele Frauen links und rechts an den Händen irgendwelche Binden dran hatten. Die waren verletzt. Der Tagesablauf war, wir haben von morgens bis abends, um 21 Uhr wurde das Licht ausgemacht, wir haben nichts gemacht. Wir waren froh, wenn die Tür aufging und wir Untersuchungen hatten, dass wir mal so halbwegs diese Krankenhausluft schnuppern konnten. Ich erinnere mich an ganz saure Gerüche und so. Ich erinnere mich an viel Medikamentengeruch. Wir haben nichts gemacht. Wir haben den ganzen Tag auf den Betten gesessen. Und dass wir da durchgedreht sind, ist doch völlig normal. " ${ }^{18}$ Eine andere Zeitzeugin bestätigt diese Aussage: „[W]ir hatten nichts zu tun. Wir durften etwas lesen. Da gab es ein paar Bücher."Kontakte zur Außenwelt wurden in den drei Wochen des Aufenthalts unterbunden. Bis auf eine Ausnahme gab es „keinerlei Außenkontakte. (...) Da gab es noch etwas Besonders übrigens, ab und zu kam eine Fürsorgerin, so wurde uns die vorgestellt, und der Kontakt nach außen war ja völlig abgeschnitten, keine Besuche, keine Post, gar nichts und die hat mir aber einen Brief mitgebracht von meiner Mutter, so als Ausnahme. Sie kannte auch den Inhalt und hat mir dann auch noch einmal ins Gewissen geredet. " ${ }^{19}$ Arbeitstherapeutische Beschäftigungen wie sie in Halle (Saale) Alltag waren oder pädagogische Ansätze wurden in der geschlossenen Venerologischen Station von Berlin-Buch nicht verfolgt. Die alltägliche Monotonie wurde lediglich durch drei Ereignisse unterbrochen: Gynäkologische Untersuchungen, gewalttätige Übergriffe und die Einnahme von Essen, das von den Pflegerinnen mit einem Essenwagen auf die Station gebracht und dort ausgeteilt wurde.

Die täglich stattfindenden gynäkologischen Untersuchungen inklusive des Abstrichs wurden von einer Pflegerin durchgeführt. Ärzte ließen sich kaum auf der Station blicken. Wie in Halle (Saale) mussten die Zwangseingewiesenen morgens in einer Schlange stehen und warteten auf die Untersuchung: „[W]ir mussten halt anstehen und da gab es so ein Glas mit, ich muss irgendwie gefragt haben, aus welchem Material das ist, aus Platin. Das war wie eine Schlinge, also oben so, ich kann es schlecht sagen, welchen Durchmesser, ein paar Millimeter Durchmesser, hohl, irgendwie eine Schlinge und dann noch ein Stück Metall dran und unten halt ein Griff. Ja, das war das Untersuchungsinstrument sozusagen. Ja und da war dann so ein, ich glaube, ein Bunsen-

517 Interview mit Frau BPA, geführt von Florian Steger und Maximilian Schochow, Halle (Saale), den 28. Oktober 2014. 518 Interview mit Frau BPB, geführt von Maximilian Schochow, Halle (Saale), den 15. September 2015.

519 Interview mit Frau BPA (Anm. 517). 
brenner, jedenfalls musste man das immer drüber halten, sollte zum Desinfizieren sein und ja, dann ging es halt nacheinander hinter den Vorhang und da wurde dann ziemlich brutal die, also diese Frauenarztinstrumente, die so einen Knick haben, ich weiß nicht, wie die heißen, wurden dann halt eingeführt und wenn man gejammert hat, wurde man noch beschimpft, mit den Männern tut es ja auch nicht weh und solche Dinge halt." Der tägliche Abstrich wurde „mit dieser Schlinge wohl genommen. Du konntest ja selber nicht sehen und ich überlege jetzt schon die ganze Zeit, ich bin mir einfach nicht sicher, aber ich tendiere dazu, dass auch in die Harnröhre mit diesem Instrument reingegangen wurde, ja. " 520

Neben den groben Untersuchungen machten viele Zwangseingewiesene Erfahrung mit Gewalt, die von anderen Patientinnen ausging: „Und die Frau neben mir, die hat mich gefragt, ob ich ihr helfe, die anderen schlagen sie immer. Und ich habe total Angst bekommen, dass es mich auch trifft." Obwohl die Frau niemanden provoziert hatte, wurde sie aus dem Zimmer geholt und mit der „Toilettenbürste verprügelt“. Der Überfall „war ganz willkürlich, wenn man die als Opfer auserwählt. Ich weiß nicht, die Hierarchie hat da wohl schon eine ganze Weile geherrscht. Es hörte ja dann auch auf, zwei, drei Tage später, als die beiden Anführerinnen dort entlassen wurden. " Die Gewalt ging von Frauen an Frauen aus. „Ich muss dazu sagen, also ich höre die Schreie noch von der Frau, aber ich bin auch schwer traumatisiert aus meiner Kindheit, was körperliche Gewalt im Elternhaus betrifft und deswegen habe ich das bis jetzt noch gar nicht hochgeholt diese Situation, ja. Und diese Hilflosigkeit habe ich natürlich dann auch wieder gefühlt, ja. Die Frau hat mir leid getan und hatte auch nicht den Mut, da irgendwas zu machen. " ${ }^{21}$

Die Aggressionen der Zwangseingewiesenen richteten sich aber nicht nur gegen andere Frauen, sondern auch gegen die Institution. „Wir wollten aber unbedingt ein Loch drinnen haben. Also es muss ja einen Besen gegeben haben, es war ein Besen oder ein Stiel, keine Ahnung. Und das haben wir halt zusammen geplant, ja. Und uns auch natürlich gefürchtet vor irgendwelchen Repressalien von den Schwestern, wobei ich mir aber überhaupt gar nicht, ich kann mich an nichts erinnern, was einen direkt jetzt geblüht hätte oder so, ja. (...) Nein, weil diese Scheibe da kaputt zu machen, war ja sicherlich schon was Besonderes dort und ich kann mich nicht erinnern, dass das irgendwie Repressalien zur Folge hatte. "Bestrafungen oder Belobigungen für gutes oder schlechtes Verhalten vonseiten des Pflegepersonals oder der Ärzte, wie in Halle (Saale) üblich, gab es in Berlin-Buch nicht. Dies bestätigt auch eine Zeitzeugin, die 1975 auf der Station war: „Und ich weiß noch, dass ich, dass wir waren glaube ich drei Frauen oder Mädchen, Mädchen waren wir ja noch, wir haben uns angefreundet und dann haben wir versucht, von der Schwester 
Streichhölzer zu bekommen. Dann haben wir versucht, unsere Betten anzuzünden und dann haben wir natürlich doch Panik gekriegt; haben das ausgemacht, weil es so gestunken hat. Wir haben ja Monate, Wochen haben wir keine frische Luft bekommen. "Repressalien folgten keine. ${ }^{522}$

In einem Bericht aus dem Jahr 1977 heißt es zu solchen und weiteren Vorfällen: „Es kam fortlaufend zu Zerstörungen der Fenstersicherungen, Ausstiegen per Bettlaken und Zertrümmerungen des Mobiliars. Alle zerstörten Gegenstände wurden als Tatwerkzeuge für weitere Zerstörungen genutzt. Erst nachdem 4 schwere Verletzungen einschließlich Querschnittslähmungen und Knochenbrüchen aufgetreten waren, konnten Fenster-Innengitter angebracht werden." Betten, Tische, Nachtschränke, Hocker und Stühle wurden demoliert. „Derzeitig besitzen die Insassen in der Wand verankerte Liegen, die auch noch aus ihren Befestigungen herausgerissen werden. Selbst 80 kg schwere Innengitter der Fenster werden aus den Verankerungen gerissen und deren Schlösser zerstört." "523 In der Analyse der Ursachen für diese Gewaltausbrüche wurden vom Chefarzt der Station im Kern vier Aspekte hervorgehoben: 1. der soziokulturelle Hintergrund der Zwangseingewiesenen, 2. die mangelnde Ausbildung des medizinisch-pflegerischen Personals, 3. die Unterbesetzung der Station mit Fachkräften und 4. eine fehlende einheitliche Ordnung.

Zum soziokulturellen Hintergrund der Zwangseingewiesenen heißt es etwa im Papier von Elste, dass es sich „bei der Mehrzahl der Insassen um asoziale Elemente, Personen mit gemindertem Bildungsgrad, teils sogar kriminellen Erfahrungen, schlechten Erfahrungen aus Kinderheimen, Jugendwerkhöfen, Jugend-Strafanstalten und Hafteinrichtungen oder sogar um notorische Ausbrecher aus diesen Einrichtungen handelt". Um diese Personen zu disziplinieren und vor allem ihr Gewaltpotential zu zügeln, hätte es speziellen Personals bedurft. Doch dieses Personal stehe nicht zur Verfügung. Die medizinischen Fachkräfte würden „über keinerlei Ausbildung auf diesen Gebieten verfügen, bestimmte Schwierigkeiten u.a. auch in Fragen des Rechtssicherheit "wären offen. Darüber hinaus sei die personelle Unterbesetzung für die Zustände verantwortlich: „Erschwerend kommt hinzu, daß diese Abteilungen bereits seit Jahren mit stärkster Unterbesetzung arbeiten, teils auch schon wegen fehlender Mitarbeiter geschlossen werden mussten (Erfurt, Frankfurt), die medizinischen Fachkräfte selbst schon Schaden genommen haben." 524 Schließlich wird der fehlende normative Rahmen angesprochen. Zwar räume die „Verordnung zur Verhütung und Bekämpfung von Geschlechtskrankheiten“ vom 23. Februar $1961^{525}$ mit den $\mathbb{s} 20$ und 22 die Unterbringung in geschlossenen

522 Interview mit Frau BPB (Anm. 518).

523 LA Berlin: C Rep. 118 Nr. 909 1977. Reorganisation und Rekonstruktion (...). Einschätzung über Betreuung „kriminell gewordener“ (Anm. 513), S. 4.

524 LA Berlin: C Rep. 118 Nr. 909 1977. Reorganisation und Rekonstruktion (...). Einschätzung über Betreuung „kriminell gewordener“ (Anm. 513), S. 1.

525 Verordnung zur Verhütung und Bekämpfung von Geschlechtskrankheiten (Anm. 23). 
Stationen ein. „Jedoch besteht seit 1961 keine Ordnung, noch ein Status, worin festgelegt wird, wie eine geschlossene Abteilung a) zur Sicherung der Verwahrung von Kranken, Gefährdeten und Säumigen und b) zur Sicherung der Mitarbeiter vor Überfällen, Geiselnahme u.a. eingerichtet sein muß, noch besteht c) eine Ordnung über Disziplinarmaßnahmen und Umfang der sozialen Möglichkeiten für Insassen einer solchen Abteilung. " ${ }^{526}$ Dass die Aggressionen und Gewalttaten mit den willkürlichen Zwangseinweisungen, der Isolation der Patientinnen, mit der Festsetzung in einem geschlossenen Raum oder ganz allgemein mit dem System „Geschlossene Venerologische Station“ in einem Zusammenhang stehen könnte, tauchte als Gedanke in der Situationsanalyse von Elste nicht auf.

Vor dem Hintergrund dieser Situationsanalyse wurden vom Chefarzt in vier Bereichen Maßnahmen vorgeschlagen: Verbesserungen der Sicherungseinrichtungen, der Arbeitsbedingungen, der materiellen Grundlagen und schließlich der Regulierung von Schäden. ${ }^{527} \mathrm{Zu}$ den Verbesserungen der Sicherungseinrichtungen zählten unter anderem der „Umbau auf verstärktere Fenster-Innengitter“, die „Einrichtung des vorhandenen 2-Bettenzimmers als Separierungsraum“, der „Einbau einer zerstörungssicheren Krankenrufanlage“, die „Anbringung von Rauchmeldern in allen Krankenzimmern“, der „Anbau von Beleuchtungskörpern, die durch Drahtkorb gesichert sind“, die „unzerstörbare Verankerung der Schlafliegen“, der „Einbau einer festinstallierten Eßecke“ oder die „Einrichtung einer Örtlichkeit zur Gewährleistung von zeitweisen Aufenthalten an der frischen Luft“. Zu den Verbesserungen der Arbeitsbedingungen zählten die „Einrichtung von Planstellen und deren Besetzung mit speziell ausgebildeten Fürsorgerinnen“ zur ganztägigen Betreuung, die „Gewährung von Erschwerniszulagen“ für die Mitarbeiter sowie die „Fort- und Weiterbildung“ des medizinisch-pflegerischen Personals zum Umgang mit Zwangseingewiesenen einer geschlossenen Station. Unter dem Stichwort „Schaffung materieller Grundlagen“ ist aufgeführt, dass die Zwangseingewiesenen „Gegenstände der Körperhygiene“ sowie „regelmäßig die Tagespresse“ erhalten sollten. Zusätzlich sollte ein finanzieller Fonds gebildet werden, um den Zwangseingewiesenen Fahrkarten bei der Entlassung zahlen zu können. Schließlich sollten vom Justiziar des Klinikums Berlin-Buch Regularien entwickelt werden, wie künftig Schäden durch böswillige Zerstörung gehandhabt werden. ${ }^{528}$

Dieser Maßnahmenplan hatte nicht die Öffnung oder gar die Auflösung der geschlossenen Station zum Ziel. Vielmehr wurde das Ziel verfolgt, die Station

526 LA Berlin: C Rep. 118 Nr. 909 1977. Reorganisation und Rekonstruktion (...). Einschätzung über Betreuung „kriminell gewordener“ (Anm. 513), S. 1.

527 LA Berlin: C Rep. 118 Nr. 909 1977. Reorganisation und Rekonstruktion der Psychiatrischen Klinik im Städtischen Klinikum Buch. Vorschlag zur Verbesserung der Sicherungseinrichtungen in der geschlossenen Abteilung der Station 114 C der Hautklinik.

528 Vgl. LA Berlin: C Rep. 118 Nr. 909 1977. Reorganisation und Rekonstruktion (...). Vorschlag zur Verbesserung der (Anm. 527), S. 1-3. 
noch stärker abzuschotten. Die einzelnen Punkte ließen den Eindruck entstehen, hier werde ein Hochsicherheitstrakt für Straffällige geplant, der ausbruchssicher und verriegelt von der Außenwelt bestehen müsse. So war es auch nicht verwunderlich, dass in den Planungen stets auf das Haus 213 als Vorbild für die Umgestaltung der Station $114 \mathrm{C}$ verwiesen wurde. Im Haus 213 waren „kriminell gewordene Täter" und überstellte Häftlinge untergebracht, die mithilfe von Sicherungsmaßnahmen und -vorkehrungen des Strafvollzugs am „Entweichen/Ausbrechen“ gehindert wurden. Zwar war die Aufstockung des Stellenplans für Fürsorgerinnen ausgewiesen, doch eine mögliche therapeutische Begleitung durch einen Psychologen wie in Halle (Saale) oder die Einbindung der Eltern und Angehörigen war nicht vorgesehen. Vielmehr setzte der Plan auf Repression nach dem Vorbild des Strafvollzugs. Mit dem Maßnahmenpaket von Günter Elste näherten sich Ende der 1970er Jahre die Bedingungen in der geschlossenen Venerologischen Station Berlin-Buch jenen Verhältnissen an, die in Halle (Saale) seit Anfang der 196oer Jahre umgesetzt worden waren.

\subsection{Die „besonderen Vorkommnisse“ von 1977 und die Folgen für die geschlossene Venerologische Station im Städtischen Klinikum Berlin-Buch}

Von den „besonderen Vorkommnissen“ war der Minister für Gesundheitswesen Ludwig Mecklinger in einem Schreiben im November 1977 informiert und um Unterstützung bei der Umstrukturierung der Station gebeten worden. ${ }^{229}$ Die Umstrukturierungen bezogen sich vor allem auf die Verschärfung der Sicherungseinrichtungen. Anhand der Aussagen von Zeitzeugen lässt sich rekonstruieren, wie die Station nach 1977 gestaltet war und wie die Zwangseingewiesenen in den folgenden Jahren behandelt wurden.

Eine Zeitzeugin, die im August 1978 in der geschlossenen Venerologischen Station in Berlin-Buch zwangseingewiesen worden war, beschrieb ihre Einweisung und anhand einer Skizze des Grundrisses (Abb. 19) den Aufbau der Station im Sommer 1978. Sie erinnert sich: „1978 habe ich versucht gehabt mir das Leben zu nehmen und bin daraufhin erstmal ins Oskar-Ziethen-Krankenhaus gekommen in Berlin-Lichtenberg und dann in das Kinderkrankenhaus, was auch in Lichtenberg ist und habe dort erst einmal eine Woche verbleiben müssen, also eine Unterstützung bekommen, warum ich mir das Leben nehmen wollte. Habe mich dann damit beschäftigt, dass diese Kleinkinder, ja ich mit denen irgendwie Spaß gemacht habe, um mir ein bisschen die Zeit zu vertreiben. Musste allerdings auch nur in einer Strumpfhose und im T-Shirt rumlaufen und das war für mich als 15-Jährige auch schon sehr entwürdigend, sehr peinlich gewesen, ja, wie so ein großes Baby da zu laufen. Und bei Mittagstisch

529 LA Berlin: C Rep. 118 Nr. 909 1977. Reorganisation und Rekonstruktion (...). Einschätzung über Betreuung „kriminell gewordener“ (Anm. 513). 
sagte dann eine Schwester zu mir, eine ältere, dass ich jetzt woanders hinkomme, was man da nicht untersuchen kann und habe nur dann angefragt gehabt, ob da Erwachsene darunter sind, weil wie gesagt, ich bin da eine Woche nur in einem weißen Raum gewesen, also das Einzige was bunt war, war ich gewesen ja und eben diese Zeit zu überbrücken - ,Warum, wieso, wie geht's weiter?', also keinerlei Informationen, und habe mich dann erstmal gefreut gehabt, dass ja, dass sich jetzt irgendwas bewegt, aber immer mit dem Hintergrund, dass ich nicht in die Klapse kommen wollte, sondern erneut, ja, weil wie gesagt, ich habe von meiner Mutter eben halt auch nicht die besten Erfahrungen gemacht. Die haben sie eigentlich auch kaputttherapiert ja, das war auch nicht so Absicht gewesen, dass da irgendwas aufgeklärt oder aufgearbeitet wird oder ich irgendwelche Medikamente bekomme und so ja, genau. Und wurde dann abgeholt, da saß die Jugendamtstante, die saß vorne und hinten saß ein Praktikant, was für mich auch vollkommen unangenehm war. Nicht für ihn jetzt, sondern ja, jetzt werde ich irgendwo hingefahren ohne werthaltige Informationen, wo es, einfach irgendwo hingefahren und in Buch kann ich mich erinnern, da sind wir vorne rein, noch eine Etage höher und da hat eine Frau mit so einer Turmfrisur, so wie das in Goer, 7oer Jahre modern war, die Tür aufgemacht und hat gesagt, zu mir dann, dass ich ziemlich groß geworden bin und hatte lange aber ganz große Sorge gehabt, doch wieder in einer Klapse oder irgendwo gelandet zu sein und wurde dann mehr oder weniger übergeben. " In der ersten Etage war eine große Stahltür. Hinter dieser Tür öffnete sich ein kleiner Raum, in dem Reinigungsgeräte standen und dann kam ein Citter, welches den Raum begrenzte. Hinter dem Gitter öffnete sich ein langer Flur, von dem rechts das erste Zimmer abging. In dem ersten Zimmer befanden sich sechs Betten, ein Tisch, ein Waschbecken und zwei mit einem Stahlgerüst vergitterte Fenster. „Die Gitter waren davor gewesen, also richtig monströse Gitter. (...) Und die Fensterscheiben waren mit diesem dicken, also es war ein ganz dickes Glas mit so Draht gewesen. Du konntest aber auch nicht irgendwas aufmachen oder so. Die ganzen Wände, die waren alle vollgeschmiert gewesen mit irgendwelchen Sprüchen und wann man entlassen wird, wie oft man da war, wo der Jesus lebt, Allah auch und andere." Ging man den langen Flur weiter, kamen links zwei Fenster in „den Hof“. Am Ende des Flures „war das WC gewesen. Das war so ein ganz längliches, war aber irgendwie da hinten war eine Toilette und dann nur ein Waschbecken. "Im Bad war ein Fenster und im Zentrum des Raumes ein Abfluss in den Boden eingelassen. Ging man nach rechts um die Ecke, schloss sich ein langer Flur an, von dem links zwei weitere Zimmer abgingen. Ein „ganz kleines Zweierzimmer“ und dann ein Vierbettzimmer. Die Zimmer hatten jeweils „ganz kleine Fenster.“ Das kleine Zweibettzimmer und das Vierbettzimmer hatten jeweils ein Waschbecken. Das Zweibettzimmer war ,in der Zeit abgeschlossen gewesen“ und das Vierbettzimmer „wurde dann mehr oder weniger geschlossen“, da nicht alle Betten benötigt wurden: „Also zu meiner Zeit war es nicht voll belegt. “530

530 Interview mit Frau BPC, geführt von Florian Steger und Maximilian Schochow, Halle (Saale), den 3. Dezember 2014. 


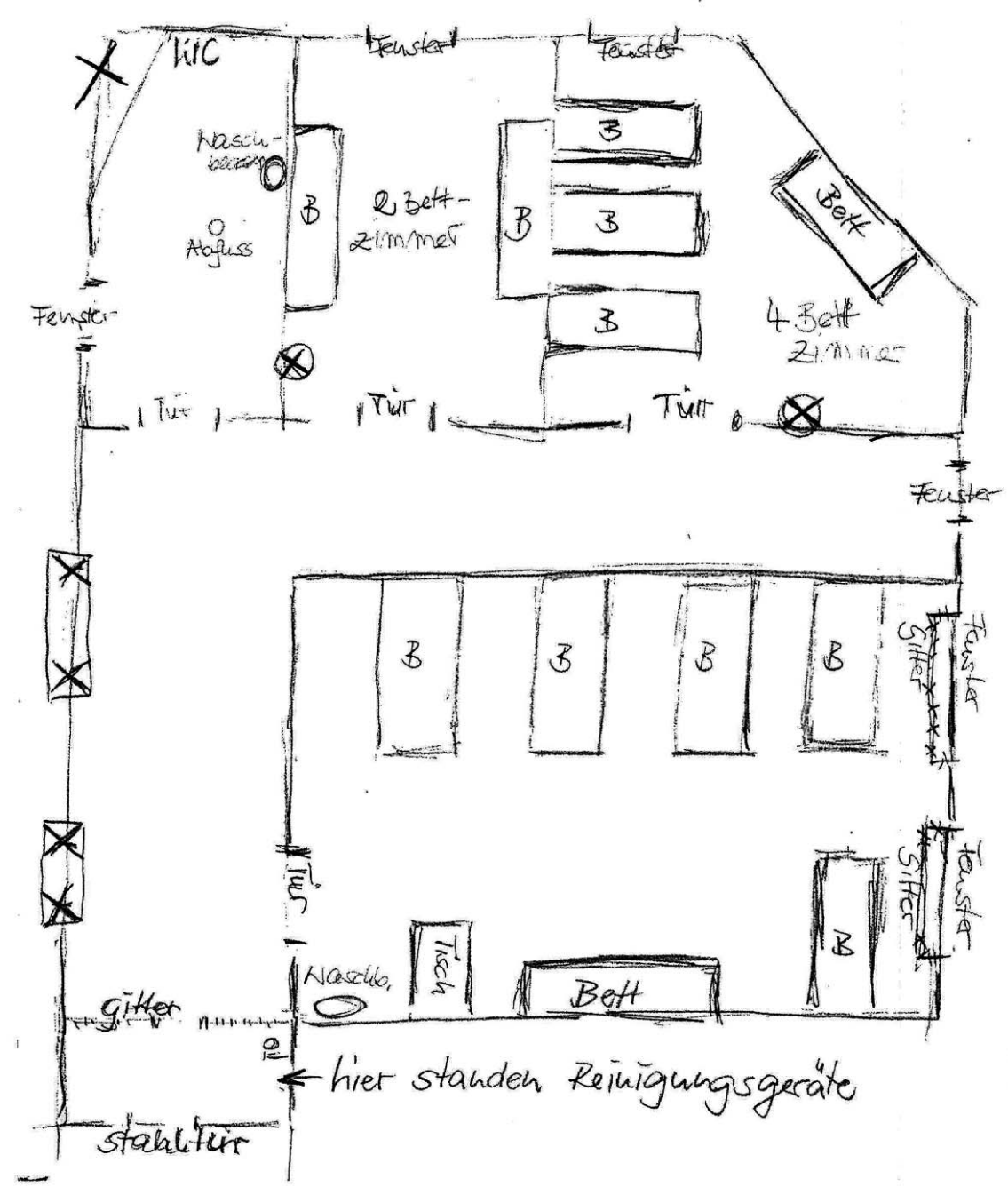

Abb. 19 Grundriss der Station 114 C der geschlossenen Venerologischen Station in Berlin-Buch (Rekonstruktion 2014)

1978 gestaltete sich der Alltag auf der Station wie folgt: „Also die gängige Praxis war gewesen, dass du morgens, also in aller Herrgottsfrühe, wie gesagt, rausgeholt worden bist." Anschließend gingen die Frauen geschlossen in den offenen Bereich, wo sich das Untersuchungszimmer befand. Vor dem Untersuchungszimmer mussten sich die Frauen anstellen und auf die tägliche gynäkologische Untersuchung warten. Das Untersuchungszimmer war ein verwinkeltes Zimmer. Die „Hälfte war Schwesternzimmer, (...) das war nochmal ein ganz kleiner Raum gewesen, so ein Eckraum, da war der Untersuchungs- 
6.4 Die „besonderen Vorkommnisse“ von 1977 und die Folgen für die geschlossene Venerologische Station im Städtischen Klinikum Berlin-Buch

raum gewesen. Und wir mussten auch immer draußen eben halt warten und dann wurde immer ein Abstrich gemacht. (...) Das war eine ganz gängige Praxis gewesen, dass du jeden Morgen dorthin kommen musstest und dann gab es eben auch die Medikamente abends in der Austeilung. "Nach der morgendlichen Untersuchung gab es auf der geschlossenen Station Frühstück. ${ }^{331}$

Weitere Beschäftigung gab es nicht. Die Zwangseingewiesenen arbeiteten weder auf der Station noch auf dem Gelände des Klinikums. Letztlich waren sie den ganzen Tag auf dieser Station in ihrem Zimmer. Abwechslung bot lediglich der Tabakkonsum: Zum Nachmittag wurden die Raucherinnen manchmal ,in diesen länglichen Raum reingeholt, da wo auch Besucherraum war, so ein Allgemeinraum und da kann ich mich noch entsinnen, da war auch alles Grünfläche gewesen, wo wir aus dem Fenster geguckt haben und die ganzen Ärzte sind darauf vorbeigegangen und klar, ich habe dann eben halt immer auch nur den Affen dort gespielt ja, also um irgendwie eine Aufmerksamkeit zu bekommen. (...) Na wie viel haben wir geraucht - vielleicht drei alle, weil, man hat ja immer alle eingeladen und dann war es auch schon durch gewesen." Zigaretten waren eine begehrte Ware in der geschlossenen Station, die nur über das Personal oder über Androhung von Gewalt organisiert werden konnte. „Na Zigaretten konnten wir da nicht haben, also wenn, da haben wir, das war diese subtile Gewalt gewesen ja, diese feinfühlige Gewalt." ${ }_{532}$

Viel hatte sich zwischen 1977 und 1978 nicht verändert. Sicher ist, dass der Ausbau der Sicherungseinrichtungen zumindest teilweise durchgeführt wurde. Dafür sprechen sowohl die Gitter an den Fenstern als auch die Stahlrahmen, welche die Gitter fassten. Damit sollte ein Herausbrechen der Gitterstäbe aus dem Mauerwerk verhindert werden. Auch deutet vieles auf eine gewisse Umgestaltung der Station hin. Der Einbau eines Waschbeckens in dem kleinen Zweibettzimmer beispielsweise und die Aussage, dass dieser Raum verschlossen war, deuten darauf hin, dass dieses Zimmer tatsächlich in einen Separierungs- und Isolierungsraum umgebaut wurde. Darüber hinaus wurden die Abläufe geändert. Die Zeitzeugen, die vor 1977 auf der Station waren, beschrieben, dass die gynäkologischen Untersuchungen auf der geschlossenen Station in einem abgetrennten Bereich des Flures stattfanden. Für das Jahr 1978 steht die Aussage, dass die Untersuchungen im Bereich der offenen Station durchgeführt wurden. An Details, wie etwa mit Drahtgeflecht abgeschirmte Lampen oder Rauchmelder, können sich die Zeitzeuginnen in den Interviews nicht erinnern. Möglicherweise waren die Umbauarbeiten 1978 noch nicht abgeschlossen und weitere Eingriffe in die bauliche Struktur folgten zu einem späteren Zeitpunkt.

Offenkundig ist vielmehr die Kontinuität, mit der die einmal entwickelten Strukturen bestanden: Die Frauen wurden nicht, wie 1977 geplant, ganztägig

531 Interview mit Frau BPC (Anm. 530).

532 Interview mit Frau BPC (Anm. 530). 
von einer Fürsorgerin betreut. Im Gegenteil blieben sie sich nach wie vor allein überlassen. Auch wurden ihnen keine Beschäftigungen angeboten - weder verpflichtend, noch fakultativ. Wie ein roter Faden zieht sich die Beschäftigungslosigkeit und Untätigkeit durch den Alltag der Zwangseingewiesenen. Dies gilt auch für die Prozedur der Aufnahme in die geschlossene Venerologische Station. Eine Frau, die 1975 zwangseingewiesen wurde, erinnert sich, dass sie sich entkleiden und einen Krankenhauskittel anziehen musste: „Auf jeden Fall irgendwann, also diese Blutabnehmerei, die hat mich dann wahrscheinlich sehr beschäftigt. Und auch gewogen, gemessen, ja, Daten wurden aufgenommen und dann habe ich noch eine Zahnbürste bekommen und ein Stück Seife und dann sind wir einen Flur lang gegangen. " 533 Aus der Perspektive einer 1978 eingewiesenen Frau verlief die Einweisung wie folgt: „So und dann habe ich auch in so einem großen Raum, also so in etwa hier gesessen und dann kam so eine kleine Ärztin, also so eine kleinere und hat mir ganz doofe Fragen gestellt, wie ob ich einen Freund habe, wann ich das erste Mal Sex hatte, (...) ob ich schon mal Selbstbefriedigung gemacht habe und da ist mir dann die Hutschnur geplatzt und habe sie gefragt gehabt, ob sie jemanden kennt, der es noch nicht gemacht hat. (...) Und dann kam so eine etwas Ältere, so aus der Zopfzeit auch übrig gebliebene ältere Dame mit Kittel und die hat mir dann so einen grünen Bademantel, Badelatschen, also auch schon getragen und dann auch so ein Nachthemd, und ich musste mich dann eben halt ausziehen und sie hat mich dann den langen Flur wieder mitgenommen und nach links rein und dann war hier so eine Stahltür gewesen und die hat sie dann aufgeschlossen." ${ }_{534}$

Auf der geschlossenen Venerologischen Station in Berlin-Buch wurden zudem neue Kosmetikprodukte getestet. Bereits 1964/65 war die Hautklinik des Städtischen Krankenhauses Berlin-Buch umgebaut worden, sodass sich zusätzliche Spezialisierungen entwickelten. So entstanden unter anderem eine Kosmetikabteilung, ein größeres klinikspezifisches Laboratorium oder eine Kinderstation. ${ }^{535}$ Kosmetikartikel gehörten seit der „Zweite[n] Durchführungsbestimmung zum Arzneimittelgesetz - Gesundheitspflegemittel" 536 vom 15. Mai 1964 zu den Gesundheitspflegemitteln. Laut dieser Durchführungsbestimmung musste die Unbedenklichkeit in der Anwendung dieser Gesundheitspflegemittel sichergestellt sein. In welcher Form das geschehen sollte, war hier nicht geregelt.

Unabhängig voneinander berichten die Zeitzeuginnen übereinstimmend, dass an ihnen Tests durchgeführt wurden. Eine Zeitzeugin, die 1975 in Berlin-Buch zwangseingewiesen wurde, erinnert sich: „Es war ja die geschlossene Station

533 Interview mit Frau BPA (Anm. 517).

534 Interview mit Frau BPC (Anm. 530).

535 Wolff, Kalinich: Zur Geschichte der Krankenhausstadt Berlin-Buch (Anm. 488), S. 234.

536 Zweite Durchführungsbestimmung zum Arzneimittelgesetz - Gesundheitspflegemittel - vom 15. Mai 1964.

In: Gesetzblatt der DDR. Teil II, Nr. 56, S. 502-504. 
und die haben ja auch Kosmetik an uns getestet, die noch nicht auf dem Markt war. (...) Man hat uns auch gesagt, dass, als wir gefragt haben, wofür das ist: ,Ja, das wird jetzt getestet, das kommt demnächst, das ist so eine neue Jugendserie, mit irgendwelchen Pickelabdeckstiften und so weiter. Das haben die alles bei uns draufgeschmiert und dann immer mal geguckt, wie sich die Dinge entwickeln." ${ }^{37}$ Eine weitere Zeitzeugin, die ebenfalls 1975 zwangseingewiesen war, beschreibt die Situation wie folgt: „Sie kamen mit so einem riesengroßen, ich sage mal, das war wie so diese riesengroßen Eisenwagen, wo die so Essen draufgemacht haben und da waren so vier bis fünf Paletten drauf mit Schminke. Und da drunter war nochmal eine große Palette und da hatten die so Stifte, so Kajalstifte drauf." Aufgeklärt über die Tests an sich oder die möglichen Nebenwirkungen wurden die Zwangseingewiesenen nicht. Die Kosmetikprodukte wurden durch das medizinisch-pflegerische Personal ohne Aufklärung und Einwilligung aufgetragen: „Die haben nur zu uns gesagt: ,Wir wollen die Kosmetik ausprobieren. ' Und die haben uns das so auf eine charmante Art beigebracht oder erzählt, dass wir so gutgläubig da reingelaufen sind und haben gesagt: ,Okay, dann lassen wir das über uns ergehen. ““538

Für die Tests wurde den Zwangseingewiesenen ein Gitter auf den Rücken gemalt, was auf das Testen der Hautverträglichkeit oder auf Allergietestungen hindeutet. „Und dann wurden so Lippenstifte genommen und von jedem Lippenstift haben die, das waren so ganz kleine Stifte, schwarz, Plastik, schwarz und da waren so ganz kleine Stifte, waren das. Oh Mensch, wie groß, ja, kann ich gar nicht sagen, waren ganz kleine Stifte, waren das, und diese Kosmetik haben die täglich, haben sie dann weggewischt mit so einem Desinfizierzeug, wieder neues Kreuz drauf und dann haben sie wieder mit uns gemalt. Ich weiß, dass keine Möglichkeit bestand uns dagegen zu weigern. Die Möglichkeit bestand nicht. Die haben zu uns gesagt, das ist ein Muss, das müssen alle Mädchen machen, es passiert uns nichts, wir brauchen keine Angst haben." In einigen Fällen kam es zu Hautreaktionen. „Ich (...) erinnere mich dran, dass ich mich totgekratzt habe am ganzen Körper, habe ich dann so an die Arme, am Rücken haben wir dann Ausschlag bekommen. Das war denen auch egal. Das wurde am nächsten Tag abgewaschen, dann wurde wieder neu draufgemalt. Dann haben sie zu uns gesagt: ,Das ist nur vorübergehend, das heilt alles wieder ab; das ist nichts Schlimmes, das ist die normale Reaktion von der Schminke.“'“539 Solche Tests wurden mehrfach pro Zwangseingewiesene durchgeführt: „Also ich denke schon, dass es das ganze Zimmer war, mit Rücken freimachen, weil ich habe ja bei mir das Gitter nicht sehen können, aber bei jemand anders auf dem Rücken, ja. Also war es mindestens noch eine weitere Frau, ja." ${ }^{540}$ 
Eine Zeitzeugin berichtet aus dem Jahr 1978: „Und nach den Untersuchungen war das eine gängige Praxis gewesen, dass man dann ins Schwesternzimmer und dann sollte man seinen Rücken freimachen und dann sollte, hier solche Allergietest und dann habe ich das auch nochmal bei den Mädels nachgefragt, wie das sein kann, warum das so ist und so und habe der Schwester auch gesagt, wenn sie mich noch einmal anfasst oder mich belästigt, dann nehme ich sie mir und schmeiße sie aus dem Fenster, also wie gesagt, ich habe schon auch einmal versucht gehabt, dagegen anzukämpfen, weil ich das einfach wissen wollte. ,Was macht ihr eigentlich hier, für was ist das gut?"“ Diese Allergietests wurden also im Anschluss an die gynäkologische Untersuchung durchgeführt, indem in das Gitter auf dem Rücken „eine kleine Tinktur“ aufgetragen wurde. Dann wurde ,immer irgendwie nochmal geguckt (...), ob sich das dann entzündet oder wie auch immer. "Auch Schminkutensilien wurden an den Zwangseingewiesenen getestet. „So müsste man sich das vorstellen: Hier war das Schwesternzimmer gewesen, hier war die Schminke gewesen." Die Frauen wurden mit der zu testenden Kosmetik versorgt und „haben sogar Schminke mit in die geschlossene Station (...) mit reinnehmen können“. ${ }^{541}$ Dass solche Kosmetiktests an den Zwangseingewiesenen der geschlossenen Station auch nach 1977 noch durchgeführt wurden, obwohl sich die unfreiwilligen Probanden gegen diese Tests zur Wehr setzten, zeigt die Fragwürdigkeit der vorgeschlagenen Maßnahmen von 1977.

Die ergriffenen Sicherungsmaßnahmen zielten also nicht auf eine Verbesserung der Situation der Zwangseingewiesenen ab, sondern vor allem auf die Abschottung der geschlossenen Venerologischen Station. Sie dienten dazu, den Widerstand der Zwangseingewiesenen mit Gittern und Isolationsraum zu brechen. Innerhalb der befestigten Venerologischen Station wurden die tradierten Behandlungs- und Umgangsformen fortgeführt. Von der „Zentralstelle zur Bekämpfung der Geschlechtskrankheiten“ in der Christburger Straße zwangseingewiesen, ${ }^{542}$ mussten sich die Frauen auf der geschlossenen Venerologischen Station im Städtischen Klinikum Berlin-Buch ihrer persönlichen Sachen entledigen, sie wurden untersucht und für drei bis vier Wochen isoliert. Monotonie und Langeweile beherrschten den Alltag auf der Station, der nur von den täglichen gynäkologischen Untersuchungen unterbrochen wurde. Eine Erziehung im Sinn der Hausordnung der geschlossenen Venerologischen Station in Halle (Saale) durch Arbeitseinsätze und Disziplinarmaßnahmen ist für Berlin-Buch nicht bekannt. Selbst der Einsatz von Stubenältesten ist hier nicht nachweisbar. Statt auf solche internen Disziplinierungsmaßnahmen und -instanzen wurde in Berlin-Buch auf bauliche Veränderungen gesetzt - Einbau von verstärkten Fensterinnengittern oder unzerstörbaren Verankerungen der Schlafliegen. Hierdurch sollte der drei- bis vierwöchige Aufenthalt im Sinn einer Verwahrung der Frauen und Mädchen gesichert wer-

541 Interview mit Frau BPC (Anm. 530).

542 Interview mit Frau BPA (Anm. 517); Interview mit Frau BPC (Anm. 530). 
6.4 Die „besonderen Vorkommnisse“ von 1977 und die Folgen für die geschlossene Venerologische Station im Städtischen Klinikum Berlin-Buch

den. Für den Zeitraum der zwangsweisen Unterbringung sollte sichergestellt werden, dass alle medizinischen Versorgungsprozeduren, vor allem die Provokationstests, die auch in Berlin-Buch angewandt wurden, durchgeführt werden konnten, ohne dass die Zwangseingewiesenen sich mit dem Mobiliar gegenseitig krankenhausreif schlugen oder die Einrichtung zerstörten. 